\title{
7 Der „richtige“ Ort für den „richtigen“ Film: Aufführungsrahmen und -praktiken
}

\subsection{Kinotheater und Reklamefilm: Kritische Stimmen und „Lösungsansätze“}

\begin{abstract}
Auf Schritt und Tritt begegnet der Mensch heutzutage der Werbung in irgendeiner Form, daheim beim Empfang der Post oder beim Zeitunglesen, auf der städtischen oder ländlichen Straße, in der Tram oder Eisenbahn, warum also nicht auch im Theater und Kino? Ist doch hier wie kaum anderswo die Möglichkeit gegeben, den Interessenten in einem Augenblick zu packen, wo er nicht von anderen Eindrücken abgelenkt wird und die ihm präsentierte Werbung zur Kenntnis nehmen muß, ob er nun will oder nicht. ${ }^{507}$
\end{abstract}

Die Stellungnahme des österreichischen Werbefilmproduzenten Hans Ludwig Böhm im Fachblatt Reklame-Presse aus dem Jahr 1936 versteht sich - wie auch der Rest des Artikels - als Aufruf, den werbenden Film und seine Vorteile zu protegieren. Im Sinne des Auftrags dieses informativen Berichts blieben die Probleme, welche die Aufführung von Reklamefilmen in Lichtspieltheatern schwierig gestalteten, unausgesprochen. In den 1920er-Jahren bis in die frühen 1930er-Jahre hatten die Kinobesitzer wenig Interesse an der Einbindung werbender Filme in ihren Spielplan, was sich direkt an deren mitunter gepflogenen Vorführpraktiken zeigte, kritische Berichte in den Blättern des werbenden und kaufmännischen Fachs nach sich zog und Skeptiker auf den Plan rief.

Für die Kinobetreiber war das Hauptprogramm - vornehmlich unterhaltende Spielfilme - die Attraktion jedes Lichtspielabends. Das Publikum wollte „seine Stars“ auf der Leinwand sehen. Es verlangte nach Dramen, Komödien oder Abenteuern, an denen es für einige Stunden im Dunkeln fiktiv teilnehmen konnte. Die Werbefilme im Vorprogramm waren den Zuschauern - den Berichten der Presse zufolge - großteils lästig, da die Kinobesucher wegen des Amüsements und nicht $\mathrm{zu}$ Werbezwecken das Lichtspieltheater zu besuchen pflegten. „Geschmacklose oder gar augenfällig hervorstechende Reklame“ schädigte unter Umständen „das Renommee der Filmbühne“. 508

Um die Werbeeinschaltungen möglichst kurz zu halten, den Spielplan nicht unnötig in die Länge zu ziehen und das Publikum in seiner Erwartungshaltung nicht zu stören, tendierten die Kinobetreiber bisweilen dazu, die Werbeeinschaltungen (ob Lichtdias oder Filme) schneller als bei einer ordnungsgemäßen Vorführung durchlaufen zu lassen. Die Reklamebotschaft konnte demgemäß nicht

507 Reklame-Presse, „Filmwerbung und Werbefilm“, Nr. 2, September 1936, S. 2. 508 Der Filmbote, „Der Kinematograph im Werbedienst“, Nr. 24, 12. Juni 1920, S. 16. 
entsprechend wahrgenommen werden. Zudem blieb der Saal während der Werbevorführungen, die vor Beginn des Hauptprogramms und in den Pausen erfolgte, oftmals halb oder ganz erleuchtet. ${ }^{509}$ Die von Werbefilmproduzenten wiederholt beschworene, konzentrierte Aufmerksamkeit der Zuschauer im abgedunkelten Raum war so natürlich nicht gewährleistet.

Der Umstand, wonach der Werbeteil oftmals lange vor Beginn des Hauptprogramms (etwa 10 Minuten früher) und zwischen zwei Vorstellungen lief, führte dazu, dass immer nur ein geringer Teil der Kinobesucher die Reklame tatsächlich sah. Die Präsentation des Werbeblocks nach der Wochenschau wurde schließlich vorgeschlagen, um die sichere Anwesenheit des gesamten Publikums zu sichern. ${ }^{510}$ Kritiker merkten zusätzlich an, dass die volle Auslastung der Säle auch nicht gegeben sei. Aus Deutschland meldete man 1935, dass im Jahresdurchschnitt nur etwa 30 Prozent des Platzangebots in den Lichtbildtheatern wirklich genutzt wurden. ${ }^{511}$ Vergleichszahlen aus Österreich waren nicht zu ermitteln.

Beanstandet wurde des Weiteren auch die fehlende oder mangelhafte musikalische Begleitung der Reklamebilder. Der Einsatz von Schallplatten mit Klängen und/ oder Sprechtext war in der Stummfilmzeit bereits durchaus üblich. Beim gesprochenen Wort machte im Speziellen der Synchronismus von Bild und Ton Probleme. Als „unpassend“ und „peinlich“ empfundene Dialoge wurden ebenso von den Fachblättern der Kaufmannschaft bekrittelt und als „primitiv“ abgelehnt wie Schlager, die von „süßlich-wabbliger Stimme“ sowie „alberner Melodie und Text“ bestimmt waren. $^{512}$

Aufgrund all dieser vorgebrachten Mängel standen vor allem die Reklamebüros im Kreuzfeuer der Kritik, ${ }^{513}$ die hinsichtlich Buchung und Vorführung der Werbefilme mit den Kinobetreibern Verträge abschlossen und die ordnungsgemäße Darbietung der Werbebilder gewährleisten und kontrollieren sollten. Seitens der Werbefilmproduzenten und der Lichtspielunternehmer verstärkte sich im Verlauf der 1930er-Jahre der Unmut gegenüber den Reklamepächtern. Letzteren wurde eine völlig unstringente Geschäftsgebarung vorgeworfen. Klare preisliche Vereinbarungen blieben aus, ein permanentes Unterbieten hatte Dumpingpreise sowie die Unterverpachtung einzelner Vorführungen zur Folge. Zudem würden sie „die erteilten

509 Österreichische Reklame, „Kino-Reklame“, Nr. 7, Jänner 1928, S. 23. Verkaufspraxis, „Was zur Kinoreklame gesagt werden muß“, Nr. 7, April 1930, S. 405 f.

510 Verkaufspraxis, „Was zur Kinoreklame gesagt werden muß“, Nr. 7, April 1930, S. 405.

511 Verkaufspraxis, „Streu- und Treffmöglichkeiten in der Kinowerbung“, Nr. 8, Mai 1935, S. 484.

512 Verkaufspraxis, „Was zur Kinoreklame gesagt werden muß“, Nr. 7, April 1930, S. 406 sowie ebd., „Über die akustische Ausgestaltung der Kino-Reklame“, Nr. 10, Juli 1934, S. 601-603.

513 Auf kritische Stimmen bezüglich der Vorführung der Werbefilme in Kinotheatern folgten mitunter klare Stellungnahmen seitens der Reklamepächter. Siehe: Verkaufspraxis, „Was zur Kinoreklame außerdem gesagt werden muß!“, Nr. 11, August 1930, S. 665-669. 
Bestellungen unordentlich ausführen““. ${ }^{514}$ Die Gebarung der Reklamebüros behindere nicht nur „die Entwicklung des Werbefilms in Österreich“, sondern auch „die reklametreibende Industrie, die Kinobesitzer und die Werbefilmproduzenten“ erlitten entsprechenden Schaden. ${ }^{515}$ Der „Bund der Wiener Lichtspieltheater“ und der „Verband der Kurzfilmhersteller“, in dem sich die Produzenten von Werbe-, Industrie-, Kultur-, Lehr- und Bildungsfilmen sammelten, drangen daraufhin beim Bundesministerium für Handel und Gewerbe darauf, eine Sonderbestimmung für Reklamefilme zu erlassen. Dieser zufolge sollte das Recht zur Vorführung von Werbefilmen ausschließlich einer Zentralstelle des Bundes der Lichtspieltheater zukommen. Die Betreiber der Reklamebüros lehnten eine derartige Regelung vehement ab und wollten wie bisher „die Aufführungen durch freie Verträge auf rein kaufmännischen Grundlagen regeln, bei welchen das Kino seine Interessen technisch und kaufmännisch nach eigenem Ermessen schützen“ konnte..$^{516}$

Eine völlige Umgehung der Reklamepächter in dieser Angelegenheit war jedoch nicht möglich, da rechtsgültige und langfristig abgeschlossene Pachtverträge mit diversen Kinos vorlagen. Die Idee, ein Kontrollbüro einzurichten, das die Verteilung der Werbefilme überwachen und für die Wünsche aller Interessenten in objektiver Weise sorgen sollte, fand bei 60 Prozent der Reklamepächter Zustimmung. Für eine neue Regelung reichte dies allerdings im Oktober 1936 noch nicht aus. ${ }^{517}$ Im August des Folgejahres änderte sich die Lage. Nach langwierigen Verhandlungen wurde unter Einbeziehung aller Beteiligten ein neues Regelwerk beschlossen, das „ein Unterbieten der Preise für Diapositivvorführungen und Reklamefilme“ unmöglich machen sollte. Ein detailliertes Preisregulativ war ausgearbeitet worden, das die Lage der Kinos, die Anzahl der Vorführungen und die Länge der jeweiligen Streifen in die Berechnung einschloss. Die Filme wurden fortan „durch die Reklamebüros über die Einheitsstelle des Bundes vergeben“. Ein neu konstituierter Überwachungsausschuss, bestehend aus ,je zwei Vertretern der Reklame treibenden Firmen, der Kinobesitzer, der Reklamebüros und der Reklamefilmerzeuger“ hatte von nun an dafür Sorge zu tragen, dass „die Vorführung in den Kinos klaglos funktioniert und die oftmals berechtigten Klagen der Reklame treibenden Firmen ebenso wie die des Publikums, dass man infolge des schnellen Ablaufs der Anzeige oder dem Werbefilm kaum folgen kann, gegenstandslos werden“. 518

514 WKW, Filmakten, Paket 3173/2, M 8, „Filmkonferenz, Protokolle“, Verhandlungsschrift der 5. Sitzung der Österreichischen Filmkonferenz vom 9. Oktober 1936, S. 14.

515 WStLA, MA 104, Zl. K 1015, „Schreiben des Bundes der Wiener Lichtspiel-Theater an das Besondere Stadtamt II/3“, 14. Juni 1937.

516 Der Wiener Film, „Kampf um den Werbefilm“, Nr. 12, 23. März 1937, S. 2.

517 WKW, Filmakten, Paket 3173/2, M 8, „Filmkonferenz, Protokolle“, Verhandlungsschrift der 5. Sitzung der Österreichischen Filmkonferenz vom 9. Oktober 1936, S. 14.

518 Der Wiener Film, „Regulativ für den Reklamefilm“, Nr. 32, 10. August 1937, S. 2. 
Für die geregelte Aufführung des Reklamefilms im Lichtspieltheater war also wenige Monate vor dem „Anschluss“ Österreichs an NS-Deutschland noch eine neue Vereinbarung getroffen worden. In der Praxis hatten die werbetreibenden Unternehmen und Institutionen einen anderen Weg gewählt, um ihre Reklamestreifen im bestmöglichen Aufführungskontext zu präsentieren. Ein Großteil der Filme wurde nicht oder nicht nur in festen Kinoetablissements präsentiert, sondern in Fabriken, in Schaufenstern und Geschäftslokalen, in Schulen, an universitären Einrichtungen, in der Urania, in Restaurants, in Gemeindesälen, in Vereinen, in Wanderkinos, auf Kreuzschiffen, bei Messen, Fachtagungen oder Ausstellungen. ${ }^{519}$ Dieser bevorzugte Vorführrahmen, den die angelsächsische Forschung „nontheatrical exhibition" nennt, ${ }^{520}$ war durch mehrere Grundvoraussetzungen und Entwicklungen bedingt.

\title{
7.2 „Non-theatrical exhibition“: Der Werbefilmeinsatz außerhalb des regulären Kinobetriebs
}

\begin{abstract}
Die kürzeste für eine Tonfilmwerbung übliche und mögliche Länge dürfte bei zirka 10 Meter liegen. Mit 30 Meter (eine Minute Vorführdauer) läßt sich bereits ziemlich viel sagen oder zeigen. Mehr wie 150 oder höchstens 200 Meter werden in den seltensten Fällen vonnöten sein. Eine Überschreitung dieses Ausmaßes ist auch schon darum nicht empfehlenswert, weil der Interessent unter Umständen ermüdet oder gelangweilt wird, und ferner auch mit Rücksicht auf die fixe Vorstellungsdauer der Lichtspieltheater, da sonst die Einreihung in das Spielprogramm erschwert wird. ${ }^{521}$
\end{abstract}

Wiederum war es der Werbefilmer Hans Ludwig Böhm, der in den 1930er-Jahren über die Publikation zahlreicher informativer Artikel in Fachzeitungen für das Reklamemedium Film warb, praktische Tipps gab und auf etwaige Probleme und deren Vermeidung hinwies (wie etwa in obigem Zitat). Die „Überlänge“ eines werbenden Films konnte, wie Böhm klar darlegt, dazu führen, dass der Kinobetreiber die Vorführung desselben ablehnte. Die per Film werbenden Unternehmer suchten daher sinnvolle Alternativen, um ihre Reklamemittel zielgerichtet einzusetzen. Denn in den 1930er-Jahren wiesen in Österreich zur Aufführung kommende Werbefilme

519 Vgl. dazu: Moser, Karin: Charmant - markant - provokant: Der österreichische Werbefilm. Anfänge - Weichenstellungen - Durchbruch, in: Archiv und Wirtschaft. Zeitschrift für das Archivwesen der Wirtschaft, Nr. 4/2013, S. 179. Zimmermann, Yvonne: Neue Impulse in der Dokumentarfilmforschung, in: Zimmermann, Yvonne (Hg.): Schaufenster Schweiz. Dokumentarische Gebrauchsfilme 1896-1964, Zürich 2011, S. 19.

520 Siehe dazu: Slide, Anthony: Before Video: A History of the Non-Theatrical Film, Westport 1992.

521 Contact, „Werbung durch den Film“, Nr. 12, Dezember 1933, S. 17. 


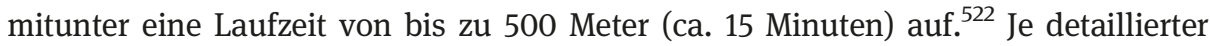
und inhaltlich sachlicher ein Film gestaltet war, umso eher bot sich eine Aufführung des Streifens außerhalb des Kinotheaters an.

Begünstigt wurde diese Entwicklung durch die Einführung, Etablierung und Verbreiterung des $16 \mathrm{~mm}$-Schmalfilms. ${ }^{523}$ Dieser war vor allem schwer entflammbar, kostengünstiger und handlicher im Einsatz als der 35mm-Normalfilm. Außerdem galten die für dieses Material konzipierten Vorführapparate als einfach zu handhaben und leicht $\mathrm{zu}$ transportieren. ${ }^{524}$ Genau solche Vorteile propagierten die Hersteller von Schmalfilmen und Schmalfilmapparaturen, um die behördliche Abwicklung und Genehmigung von Vorführungen per 8mm-, 9 1/2mm- oder $16 \mathrm{~mm}$-Film zu erleichtern. ${ }^{525}$ Befürworter fanden sie bei den Werbefilmproduzenten, den per Film werbenden Firmen sowie bei Vertretern von Bildungsinstitutionen, wobei Letztere auf eine Verbreiterung des kulturellen und wissenschaftlichen Films im Unterricht abzielten. ${ }^{526}$

Die Unternehmensvertreter betonten insbesondere die Bedeutung des ReklameSchmalfilms für die heimische Industrieförderung und forderten eine Vereinfachung der Aufführungsbestimmungen, um die Verbreitung „dieses neuen Werbemittels“ zu sichern. ${ }^{527}$ Widerstände machten sich diesbezüglich besonders in den Bundesländern bemerkbar. Die dort ansässigen Kinobesitzer fürchteten Einbußen aufgrund der vermehrt gestellten Ansuchen auf Betreiben von Wanderkinobetrieben zur Präsentation „geschäftlicher Ankündigungen“. ${ }^{528}$ Deren Opponenten (Werbefilmproduzenten, Schmalfilmerzeuger, per Film werbende Firmen) beharrten darauf, dass „geschäftliche Propaganda-Vorführungen“ keinerlei Konkurrenz für Lichtspieltheater darstellten. ${ }^{529}$ Die Handelskammern der Länder sowie das Bundesministerium für Handel und Verkehr intervenierten schließlich bei den Landesregierungen

522 Contact, „Vortrag: Wie entsteht ein Werbefilm?“, Nr. 7/8, Juli/August 1935, S. 30.

523 Vgl. dazu auch: Goergen, In filmo veritas, S. 359.

524 Verkaufspraxis, „Werbeleiter und Schmalfilm“, Nr. 8, Mai 1932, S. 473. Der gute Film, „Der Schmalfilm“, Nr. 61, 26. Jänner 1934, S. 7 f.

525 WKW, Filmakten, Paket 3173/3, M 13, Burgenländische Handelskammer, Einl. Z. B 1448/5. Ggstd.: „Schmalfilmvorführungen mit schwer entflammbaren Filmen“, September 1933.

526 ÖStA, AdR, BMHV, 581c, Grz. 93.426/33, Ggstd.: Schmalfilme. Erlassung besonderer Vorschriften, Februar-Juni 1933.

527 ÖStA, AdR, BMHV, 581c, Grz. 107.193-10/31, Ggstd.: Schwierigkeiten bei der Vorführung von Schmalfilmen in einzelnen Bundesländern, Dezember 1931.

528 WKW, Filmakten, Paket 3173/3, M 13, Wiener Allgemeine Gewerbegenossenschaft, Betreff: „Öffentliche Vorführung von Lauf- und Stehbildern“, 10. November 1932. Österreichische FilmZeitung, „Gibt es ,kinokose’ Orte in Österreich?“, Nr. 52, 24. Dezember 1937, S. 1.

529 WKW, Filmakten, Paket 3173/3, M 13, Wiener Allgemeine Gewerbegenossenschaft, Betreff: „Öffentliche Vorführung von Lauf- und Stehbildern“, 10. November 1932. Österreichische FilmZeitung, „Schmalfilm und Kinotheater“, Nr. 49, 3. Dezember 1932, S. 7 sowie ebd., „Der Schmaltonfilm“, Nr. 11, 17, März 1934, S. 3. 
dahingehend, die Regelungen für die Schmalfilmvorführungen zu lockern, da „durch eine allzu strenge Handhabung der Vorschriften gegen den ungefährlichen Schmalfilm die Interessen der in den Ländern ansässigen Unternehmern“ geschädigt würden. ${ }^{530}$

Als Vorbild galt Wien, das die feuerpolizeilichen Vorgaben für die Vorführung von schwer entflammbaren Schmalfilmen bereits 1931/32 reduziert hatte. Die Bundesländer hielten an ihren strengen Regulativen mitunter fest und verwiesen wiederholt auf „den Gegensatz von Stadt und Land und den daraus entspringenden wesentlich anders gearteten bau-, feuer- und sicherheitspolizeilichen Verhältnissen und Einrichtungen“. 531

In Wien fand die Vorführung von werbenden Filmen im Rahmen einer „nontheatrical exhibition“ weite und vielfältige Verbreitung, wie die Aktensammlung des Wiener Magistrats, Abteilung 52, der Jahre 1926 bis 1938 belegt. ${ }^{532}$ Diese Behörde hatte sämtliche Filmvorführungen in Wien im Non-theatrical-Bereich zu bewilligen und verlieh Konzessionen für Schmalfilmaufführungen. Zahlreiche Ansuchen bezogen sich auf die Präsentation von Filmen in Geschäftsauslagen, bevorzugt nach Ladenschlus ab 18 oder 19 Uhr bzw. mit Eintritt der Dunkelheit. ${ }^{533}$ Gezeigt wurden die Streifen mitunter auch über dem Eingangsbereich auf einer „Mattscheibe“534 oder in den Räumlichkeiten des Geschäftslokals. Die Bandbreite der um Vorführungskonzessionen in Schaufenstern oder in Läden ansuchenden Handeltreibenden war vielfältig. Bewilligungen erbaten unter anderem Verkaufslokale für fotografische Utensilien, ${ }^{535}$ für Radioapparate, ${ }^{536}$ für Damen-, Herren- und Sportbekleidung, ${ }^{537}$ für Seifen und Parfümerzeugnisse, ${ }^{538}$

530 ÖStA, AdR, BMHV, 581c, Grz. 107.193-10/31, Ggstd.: Schwierigkeiten bei der Vorführung von Schmalfilmen in einzelnen Bundesländern, Dezember 1931.

531 Ebd.

532 In den Verzeichnissen des WStLA wird die Magistratsabteilung 52 mit der Nummer 104 geführt. Auf allen Akten ist jedoch MA 52 vermerkt.

533 Z. B.: WStLA, MA 104, Zl. K 1236, „Konzessionsansuchen der Photohandlung Norbert Wallner“, 31. Dezember 1928.

534 WStLA, MA 104, Zl. K 709, „Konzessionsansuchen des Wirk- und Schnittwarengeschäfts von Rosa Arzt“, 14. Dezember 1927.

535 WStLA, MA 104, Zl. K 3611, „Konzessionsansuchen Photohandlung Salo Feuchtbaum“, 4. Dezember 1933.

536 WStLA, MA 104, Zl. K 1226, „Konzessionsansuchen Franz Kreuzer, Radiomaterialhandel“, 9. November 1928.

537 WStLA, MA 104, Zl. K 339, „Konzessionsansuchen der Herrenmodenfirma M. Neumann“, 15. Jänner 1927 sowie ebd. Zl. K 3143, „Konzessionsansuchen des Sporthauses Sasi“, 6. Dezember 1932.

538 WStLA, MA 104, Zl. K 2351, „Konzessionsansuchen M. E. Mayer. Parfümerie und Seifen-Fabrik“, 18. Dezember 1934. 
für Reiseangebote, ${ }^{539}$ für Süßwaren, ${ }^{540}$ für Milchprodukte, ${ }^{541}$ für Spielwaren, ${ }^{542}$ für Büromaschinen, ${ }^{543}$ für Baumaterialien und Öfen, ${ }^{544}$ für elektro-medizinische Produkte ${ }^{545}$ oder für Mineralölfabrikate. ${ }^{546}$

Bisweilen verwiesen die um eine Vorführungskonzession ansuchenden Kaufleute auf den schlechten Geschäftsgang und die allgemeine prekäre Wirtschaftslage, die erhöhte Werbemaßnahmen forderten. Die flimmernden Streifen sollten die potenzielle Kundschaft auf das Lokal aufmerksam machen und zum Verweilen einladen. ${ }^{547} \mathrm{Zu}$ diesem Zweck wurden nicht ausschließlich Reklamefilme gezeigt. Bisweilen präsentierte man auch von den Schmalfilmproduzenten eigens zur Verfügung gestellte „Leih- und Schulfilme“, die Reise- und Sportszenen ebenso im Repertoire hatten wie Lustspiele, Trick- und Marionettenfilme. ${ }^{548}$ Den Gesuchen der Händler stimmte die Behörde zu, sofern keine verkehrstechnischen Behinderungen zu erwarten waren. Bei zu schmalen Gehwegen und Verkehrsknotenpunkten vor dem Lokal sprach man sich gegen eine Vorführbewilligung aus. ${ }^{549}$ Mitunter widerrief der Magistrat Genehmigungen, etwa wenn es zu Störungen akustischer Art (Einsatz von Lautsprechern) kam oder die Sicherheitswachen die beobachtete Ansammlung einer Menschenmenge vor dem Schaufenster als gefährlich einstuften. ${ }^{550}$

Nicht selten fanden Reklamefilmaufführungen in Kaufhäusern statt. Streifen über die Kunstseidenfabrikation stellten durchaus einen Bezug zu den in den

539 WStLA, MA 104, Zl. K 3518, „Konzessionsansuchen Reisebüro Marienbrücke“, 1. August 1933. 540 WStLA, MA 104, Zl. K 3086, „Konzessionsansuchen Josef Goldstein, Vertreter der Albert Breyer Caramellenfabrik, Bregenz“, 17. Oktober 1932.

541 WStLA, MA 104, Zl. K 3197, „Milchindustrie A.G., Vorführung in Wiener Filialen“, 3. Jänner 1933.

542 WStLA, MA 104, Zl. K 21, „Konzessionsansuchen des Spezialhauses für Matador Baukasten und technische Spielwaren, Erwin Trubrig“, 12. November 1934.

543 WStLA, MA 104, Zl. K 1771, „The Rex Co., Reklamefilmvorführung-Beschwerde“, 7. Jänner 1930.

544 WStLA, MA 104, Zl. K 2101, „Konzessionsansuchen Eternit-Werke Verkaufslokal“, 13. Dezember 1937 sowie ebd., Zl. K 3094, „Konzessionsansuchen der Dauerbrandofen- und Herdfabrik, Ofengeschäft Alois Swoboda \& Co.“, 27. Oktober 1932 ebd. Zl. K 4068, „Konzessionsansuchen Hugo Hinze. Eisen- und Metallwarenhandlung“, 13. September 1934.

545 WStLA, MA 104, Zl. K 2351, „Konzessionsansuchen Karl Marholt. Röntgen- und elektromedizinische Apparate“, 5. Dezember 1930.

546 WStLA, MA 104, Zl. K 3574, „Konzessionsansuchen Shell Floridsdorfer Mineralöl-Fabrik, Schaufenster Schubertring 14“, 16. Oktober 1933.

547 WStLA, MA 104, Zl. K 576, „Konzessionsansuchen Firma Pietzner \& Fayer, Kiosk“, 9. Dezember 1927 sowie ebd., K 2971, „Konzessionsansuchen Radiohaus Horny“, 20. Mai 1932.

548 WStLA, MA 104, Zl. K 2971, „Konzessionsansuchen Radiohaus Horny“, 20. Mai 1932.

549 So z.B.: WStLA, MA 104, Zl. K 824, „Konzessionsansuchen Pfeiffers Puppenparadies“, 2. März 1928.

550 WStLA, MA 104, Zl. K 2865, Schreiben Sicherheitswache Ottakring, „Verkehrshinderung durch Vorfühurng von Laufbildern, Auslage Radiohändler Otto Löwith“, 12. September 1929. 
Räumlichkeiten feilgebotenen Waren her. Gleichfalls in diesem Rahmen präsentierte Fremdenverkehrsfilme dienten offensichtlich mehr der Entspannung und dem Amüsement der Kundschaft. ${ }^{551}$ Mitunter waren Werbefilmvorführungen Teil einer Vielzahl anderer den Kunden dargebotenen „Zusatzattraktionen“. So erklärten die Betreiber der Konditorei „Ludwig Zwieback \& Bruder“ in ihrem Gesuch mit dem Ansinnen, den Reklamestreifen Dichtl SPITzEN zu zeigen, dass ihre Räume bereits für musikalische und Tanzproduktionen, Mode-Revuen und ähnliche Veranstaltungen behördlich kommissioniert seien. ${ }^{552}$

Vereine und Genossenschaften führten ihre der Eigenwerbung dienenden Filme in den Auslagen ihrer Geschäftsstellen, in deren Versammlungslokalen, Gasthäusern und auch in Kinos vor. ${ }^{553}$ Zugleich fanden spezifische Anbieter ihre Käufer-Zielgruppe gerade in derlei Vereinigungen. Produzenten von chemischen Schädlingsbekämpfungsmitteln sowie Teer- und Asphalterzeugnissen bewarben ihre Waren per Film im „Österreichischen Verband der Kleingärtner, Siedler und Kleintierzüchter“, ${ }^{554}$ Eternit wiederum führte Filme zum Thema „Asbest“ beim „Wirtschaftsverband der Hausbesitzer Österreichs“ vor. ${ }^{555}$

Außerhalb der Kinos wurden Reklamefilme mit größerer Zielgruppenorientierung vorgeführt. Filme über Singer-Nähmaschinen präsentierte man der Genossenschaft der Schuhmacher, Streifen über Trockenplatten fanden ihr Publikum im „Österreichischen Ingenieur- und Architektenverein“. ${ }^{556}$ Reklame- und Industriewerbefilme waren oft

551 WStLA, MA 104, Zl. K 237, „Konzessionsansuchen, Vorführung der Burgenländischen Fremdenverkehrs-Schau in den Räumen des Kaufhauses A. Herzmansky“, 19. Oktober 1926. Ebd., Zl. K 1053, „, Konzessionsansuchen, Vorführung Kunstseidenstoff.-Industriefilm, A. Gerngross“, 28. August 1928. Ebd., Zl. K 2233, Konzessionsansuchen, Vorführung des Films VOM DoNAUSTRAND AUF BERGESHÖH’N, Warenhaus Gerngross, 15. Jänner 1938.

552 WStLA, MA 104, Zl. K 367, „Konzessionsansuchen, Konditorei Ludwig Zwieback \& Bruder, Reklamefilm DichTL-SPITZE“, 14. Februar 1927.

553 WStLA, MA 104, Zl. K 414, „Konzessionsansuchen Wiener Tierschutzverein, Vorführung im Schaufenster“, 16. Mai 1934. Ebd., Zl. K 1106, „Konzessionsansuchen Konsumgenossenschaft Wien, Vorführungen in Wiener Sälen und Kinos“, 4. Oktober 1928. Ebd., Zl. K 2700, „Konzessionsansuchen Konsumgenossenschaft Wien, Vorführungen in Versammlungsräumen, Gasthaussälen, Vortragssälen“, 18. September 1931. Ebd., Zl. K 443, „Konzessionsansuchen Konsumgenossenschaft Wien, Vorführungen im Genossenschaftsheim Wien XX.“, 21. September 1936. Ebd., Zl. K 4033, Konzessionsansuchen Heimbautal. Gemeinnützige Eigenheimförderungs-Genossenschaft, Vorführung von Propaganda-Filmen bei Interessenten- und Mitgliederversammlungen“, 3. August 1934.

554 WStLA, MA 104, Zl. K 797, „Konzessionsansuchen Treibacher Chemische Werke“, 13. Februar 1936. Ebd., Zl. 1731, „Konzessionsansuchen Teerag. Aktiengesellschaf für Teerfabrikate, Asphalt, Russ und chemische Produkte“, 17. Juni 1937.

555 WStLA, MA 104, Zl. K 2338, „Konzessionsansuchen Wirtschaftsverband der Hausbesitzer Österreichs, Vorführung eines Filmes der Firma Eternit“, 21. Februar 1938.

556 WStLA, MA 104, Zl. K 1863, „Konzessionsansuchen Singer Nähmaschinen AG, Vorführung des Film Die EnTSTeHUNG DER NÄHMASCHINE, 30. Jänner 1930. Ebd., Zl. K 826, „Konzessionsansuchen Medefind \& Bernfuss, Reklame-Vorträge der Firma Otto Perutz Trockenplattenfabrik, 3. April 1936. 
Teil von Propaganda-, Aufklärungs- und Bildungskampagnen. ${ }^{557}$ Das Publikum wurde etwa über die Vorteile von Elektrogeräten, über die Herstellung und den Einsatz von Wiener Gaskoks oder die Stromerzeugung umfassend per Film und Begleitvortrag informiert. Dias und Informationsblätter ergänzten das Programm inhaltlich. ${ }^{558}$

Die Vorzüge des werbenden Films im Rahmen einer „non-theatrical exhibition“ wurde von Marketingexperten und Fachmagazinen zunehmend erkannt, beworben und genutzt. So erläuterte das Blatt Verkaufspraxis 1932 den Einsatz und Nutzen des Schmalfilms wie folgt: „Ist der Bildwerfer einmal eingeschaltet, so schnurrt er ohne weitere Bedingung seinen Film herunter, und der Vertreter kann sich ganz der Erklärung und Bearbeitung der Interessenten zuwenden. Kann man die Vorteile seiner Erzeugnisse eindringlicher darlegen als durch die kurzweilige, überzeugende Wirkung eines Films, noch dazu, wenn das auf der Leinwand Gezeigte unterstrichen wird durch die individuell gefaßten Worte eines geschickten Verkäufers?“559 Ganz in diesem Sinne agierten offensichtlich auch die „Propagandisten“ der Firmen Agfa und Kodak. Bei den von ihnen durchgeführten Vortrags- und Filmabenden in Restaurants, Cafés, Hotels, Arbeiterheimen, Lehrer- oder Ingenieur- und Architektenvereinen stand die richtige Handhabung der Foto- und Filmapparate sowie die Präsentation neuer technischer Systeme im Zentrum. Unter Themenabenden wie „Eine Fahrt ins Photoreich“, „Nicht bloß knipsen - überlegt Photographieren“ [sic!] oder „Und jetzt Kunstlicht-Photos“ wurden Interessentinnen und Interessenten mit den neuen Techniken, Gerätschaften und deren Nutzung praktisch vertraut gemacht. ${ }^{560} \mathrm{Zu}$ den Vorträgen eingeladen waren vornehmlich Handelspartner (Fotound Filmhändler) sowie deren Kundinnen und Kunden. ${ }^{561}$ Präsentiert wurden Filme, deren Titel bereits auf den Inhalt schließen ließen: GeSTALTE MIT Licht, KoDACHROME,

557 Vgl. dazu auch die Ausführungen von Zimmermann, Yvonne: Dokumentarischer Film: Auftragsfilm und Gebrauchsfilm, in: Zimmermann, Yvonne (Hg.): Schaufenster Schweiz. Dokumentarische Gebrauchsfilme 1896-1964, Zürich 2011, S. 73.

558 WStLA, MA 104, Zl. K 1028, „Konzessionsansuchen Direktion der städtischen Elektrizitätswerke, Werbeveranstaltungen in Wiener Randsiedlungen“, 18. Juni 1938. Ebd., Zl. K 3541, „Konzessionsansuchen Zentral Europäische Länderbank, Niederlassung Wien - Koks-Sektion, Vorführung des Films WIENER GASKOKS vON A BIS Z in verschiedenen Lokalen“, 18. September 1933. 1938. Ebd., Zl. K 3685, „Konzessionsansuchen Zentral Europäische Länderbank, Niederlassung Wien - KoksSektion, Vorführung des Werbefilms WIENER GASKOKS im Rahmen eines Hausfrauenvortrags“, 23. Oktober 1934.

559 Verkaufspraxis, „Werbeleiter und Schmalfilm“, Nr. 8, Mai 1932, S. 474.

560 WStLA, MA 104, Zl. K 3384, „Konzessionsansuchen Agfa, Vorträge unter dem Thema „Eine Fahrt ins Photoreich“, 11. April 1933. Ebd., Zl. K 1265, „Konzessionsansuchen Agfa, 5 Vorträge unter dem Thema ,Und jetzt Kunstlicht-Photos““, 7. November 1936. Ebd., Zl. K 1483, „Konzessionsansuchen Kodak, Vorträge unter dem Thema ,Nicht bloß knipsen - überlegt Photographieren!““ [sic!], 24. Februar 1937.

561 Vgl. WStLA, MA 104, Zl. K 3619, „Konzessionsansuchen Kodak, Propaganda-Vortrag“, 20. November 1933. Ebd., Zl. K 13, „Konzessionsansuchen Agfa, Vorführung von Schmalfilmen“, 11. Jänner 1935. 
WER PHOTOGRAPHIERT, HAT MEHR VOM LEBEN: WER FILMT, DER HAT DAS LEBEN, ACH HÄTT ICH DOCH. ${ }^{562}$ Oftmals zeigte man bei den Veranstaltungen aber auch Landschaftsaufnahmen und Sehenswürdigkeiten aus Österreich, die unter Beweis stellen sollten, welch hochqualitative Aufnahmen mit $8 \mathrm{~mm}$ - oder 16mm-Apparaturen möglich waren. ${ }^{563}$ Einher gingen die Vortragsabende gelegentlich mit der Ausstellung von Kameras, Schmalfilm-Kinogeräten und „Meisterarbeiten“ der Fotografie. ${ }^{564}$

Umfassende, möglichst attraktiv gestaltete Werbeabende waren bei einigen renommierten Markenunternehmen in den 1930er-Jahren bereits üblich geworden. Die Firma Siemens \& Halske führte während der Wintermonate in verschiedenen Städten mit den ortsansässigen Händlern regelmäßig Veranstaltungen durch. Dafür wurde jeweils ein Saal angemietet, in dem die Kaufmänner eigene Präsentations-und Verkaufsstände einrichteten. Der Abend war abwechslungsreich zusammengestellt: Schallplattenmusik, Werbe- und Kulturfilme und Vorträge wurden geboten. In den Pausen konnten die potenziellen Käufer die Verkaufsstände besichtigen und sich informieren. Im Zuge einer Verlosung hatten die Besucher die Chance, elektrische Haushaltsgeräte zu gewinnen. Als Los dienten die Eintrittskarten, auf denen die Adressen der Anwesenden vermerkt waren. Letztere gingen wiederum an die Händler zurück, welche die Anschriften in ihre Interessentenkartei aufnahmen. ${ }^{565}$

Eine Vielzahl der Präsentationen im Rahmen der „non-theatrical exhibition“ richtete sich direkt an eine weibliche Zielgruppe. Während die Feinwaschanstalt Habsburg bei eigenen Filmabenden über ihren professionellen Wäschereibetrieb aufklärte, ${ }^{566}$ betrieb das Unternehmen „Persil-Gesellschaft Henkel \& Voith m.b.H.“ in Wien seit 1928 umfassende Aufklärungsaktionen, die neben Filmaufführungen Hausfrauen auch praktische Waschvorführungen bot. ${ }^{567}$

562 WStLA, MA 104, Zl. K 1125, „Konzessionsansuchen Kodak, Vorträge“, 22. September 1936. Ebd., Zl. K 1265, „Konzessionsansuchen Agfa, 5 Vorträge unter dem Thema „Und jetzt KunstlichtPhotos“, 7. November 1936.

$563 \mathrm{Zu}$ sehen waren etwa Schmalfilme mit folgenden Motiven: „Stadtpark (Wien)“, „Eislaufverein (Wien)“, „Ringstraße“, „Innere Stadt (Wien)“, „Umgebung von Wien“, „Gebirgsaufnahmen aus den österreichischen Alpen“, „Millstätter See“, „Aufnahmen vom Zug aus. Westbahnstrecke“, „Gesäuse“, „Wanderungen und Landschaften in den Tauern“, „Sommerlager am Schwarzensee“. Siehe dazu: WStLA, MA 104, Zl. K 3619, „Konzessionsansuchen Kodak, Propaganda-Vortrag“, 20. November 1933. Ebd., Zl. K 3686, „Konzessionsansuchen Kodak, Vortragsabend“, 16. Dezember 1933. Ebd., Zl. K 161, „Konzessionsansuchen Kodak, Propaganda-Abend“, 2. April 1935. Ebd.,, Zl. K 3619, „Konzessionsansuchen Kodak, Werbevorträge“, 13. Februar 1936.

564 WStLA, MA 104, Zl. K 1125, „Konzessionsansuchen Kodak, Vorträge“, 22. September 1936.

565 Verkaufspraxis, „Durch Unterhaltung werben!“, Nr. 5, Februar 1933, S. 286.

566 WStLA, MA 104, Zl. 148, „Konzessionsansuchen Habsburg Feinwaschanstalt, Filmvorführung“, 22. März 1935. Ebd., Zl. K 901, „Konzessionsansuchen Habsburg Feinwaschanstalt, Filmvorführung“, 15. Mai 1935.

567 Wiederholt vorgeführt wurde der Stummfilm VoM WASCHEN UND VON DER WÄSCHE (D 1929). Die Veranstaltungen fanden vornehmlich in Gastwirtschaften und Hotels statt. Vgl.: WStLA, MA 104, Zl. 2920, „Konzessionsansuchen Persil, Filmvorführung und Vortrag“, 12. April 1932 sowie unter 
Lebens- und Genussmittelproduzenten konnten neben Vortrag und Filmvorführung auch Verköstigungen anbieten, was in Zeiten der Wirtschaftskrise die Zahl der Interessentinnen und Interessenten entsprechend zu erhöhen vermochte. Neben der Firma „Kathreiner A.G.“, die diese Werbemaßnahme zur Anpreisung ihres Kaffeesortiments unter anderem in Wiener Gasthöfen und Bildungsanstalten nutze, ${ }^{568}$ trat hier vor allem die „Julius Maggi Gesellschaft m.b.H.“ in Erscheinung. Der Schweizer Betrieb hatte seit 1925 eine Konzession für Filmvorführungen in Wien inne, ${ }^{569}$ in der Eidgenossenschaft wurden vom Unternehmen bereits seit 1905 Wanderwerbezyklen abgehalten, damals noch unter dem Einsatz von Reklamedias. ${ }^{570}$ In der Alpenrepublik präsentierte sich Maggi als österreichisches Unternehmen. Plakate, die für „Maggi-Würze“ oder „Maggi-Rindsuppenwürfel“ warben, trugen oftmals den Schriftzug „Österreichisches Erzeugnis“, wobei mitunter die Stadt Wien (erkenntlich durch den Stephansdom) oder die Maggi-Fabrik in Bregenz (ein Tochterunternehmen des Konzerns) den Hintergrund dominierten. ${ }^{571}$

Auch bei den in Gasthäusern, Spitälern, Koch-, Haushaltungs-, Fleischselcherund Hebammen-Schulen, Waisenhäusern, Versorgungshäusern (Alten- und Pflegeheimen), Klöstern, bei der Katholischen Frauenorganisation, bei den Kinderfreunden sowie bei Sozialdemokratischen Bezirksorganisationen abgehaltenen Vorträgen setzte man auf Filme mit einem Bezug zu Österreich. ${ }^{572}$ Vorgeführt wurden die Streifen EIN BESUCH DER MAGgI-WERKE IN BREGENZ SOWIE EINE REISE VON WIEN BIS BREGENZ. ${ }^{573}$ In den präsentierten Filmen warb man für „Maggis Suppenwürze“, „Maggis Rindsuppenwürfel“ und „Maggis Suppen in Päckchen“. ${ }^{574}$ Anders als in der Schweiz, wo

dem gleichen Betreff: Ebd., Zl. K 3107, 11. November 1932. Ebd., Zl. K 3212, 12. Jänner 1933. Ebd., Zl. K 3372, 8. April 1933.

568 Siehe etwa: WStLA, MA 104, Zl. 35, „Konzessionsansuchen Kathreiner A.G., Filmvorführung“, 18. Jänner 1935 sowie unter dem gleichen Betreff: Ebd., Zl. K 905, 14. April 1936. Ebd., Zl. K 1255, 3. November 1936.

569 Vgl. dazu folgenden Akt: WStLA, MA 104, Zl. 1391, „Stellungnahme des Unternehmens Maggi zur Frage der Zulässigkeit der Vorführung von schwer entflammbaren Normalfilmen in Lokalen ohne eigenem Bildwerferraum“, 3. Februar 1937.

570 Zimmermann, Yvonne: Industriefilme, in: Zimmermann, Yvonne (Hg.): Schaufenster Schweiz. Dokumentarische Gebrauchsfilme 1896-1964, Zürich 2011, S. 286.

571 Vgl. z. B.: Contact, „Beispiele aus der Anzeigen-Werbung der Maggi-Gesellschaft“, Nr. 5, Mai 1934, S. 16-18. Österr. Reklame-Praxis, „Plakatpräsentation“, Februar/März 1936, S. 53-56 und 22 f.

572 WStLA, MA 104, Zl. K 318, „Ankündigung. Maggi-Filmvorträge“, 28. Dezember 1926 sowie unter dem gleichen Betreff: Ebd., Zl. K 348, 26. Jänner 1927. Ebd., Zl. K 799, 15. Februar 1928. Ebd. Zl. K 1602 und K 1710, 27. Jänner 1930. Ebd., Zl. K 3285, 27. Februar 1933.

573 WStLA, MA 104, Zl. K1696, „Ankündigung. Maggi-Filmvorführung“, 2. November 1929 sowie unter dem gleichen Betreff: Ebd., Zl. K 1602 und K 1710, 12. Februar 1930.

574 WStLA, MA 104, Zl. K 1391, „Stellungnahme des Unternehmens Maggi zur Frage der Zulässigkeit der Vorführung von schwer entflammbaren Normalfilmen in Lokalen ohne eigenen Bildwerferraum“, 3. Februar 1937. 
Maggi auch in Schulen auftrat und Kindern Werbe-, Kultur- und Industriefilme vorführte, ${ }^{575}$ beschränkte sich diese Tätigkeit in Österreich auf die „Erwachsenenbildung“. Im März 1938 sprach sich sogar der Wiener Stadtschulrat gegen die Vorführung von Maggi-Werbefilmen im Unterricht aus. ${ }^{576}$

Den Untersuchungen von Yvonne Zimmermann zufolge stellte das Unternehmen im Zuge der Wandervorträge in der Schweiz Suppenproben zur Verfügung, während die Lichtbilderserien und Filme von einem populärwissenschaftlichen Vortrag begleitet wurden. ${ }^{577}$ Genauere Details zum Ablauf der Veranstaltungen in Österreich liegen nicht vor. Es ist jedoch davon auszugehen, dass die Vertreter die Firmenstrategie auch hier fortführten, wenn auch mit einer speziellen Ausrichtung auf den alpenländischen Markt. National-regionale Elemente kamen hier durchaus, wenn auch in einer österreichischen Variante, zum Tragen.

Die Vielfalt der möglichen Aufführungsorte im Rahmen der „non-theatrical exhibition“ war scheinbar grenzenlos. Maggi-Filme kamen etwa auch im Wiener Landesgericht II vor Sträflingen und dem Hauspersonal zur Vorführung. ${ }^{578}$ Zeiss-Ikon und Kodak präsentierten ihre Werbefilme unter anderem im Militärkasino, ${ }^{579}$ und die „Städtischen Elektrizitätswerke“ richteten in ihren Räumlichkeiten in der Mariahilfer Straße 41 ein eigenes Reklamekino ein. ${ }^{580}$ Die Firma Titze (Kaffeeproduzent) führte einen zu Beginn der Tonfilmära eigens produzierten Klangwerbefilm in fahrbaren Werbeautos vor, die in „Höfen größerer Gebäudekomplexe der Gemeinde Wien" bei Einbruch der Dunkelheit Station machten. ${ }^{581}$

Als besonders kreativ erwies sich die Tourismusbranche bei der Auswahl ihrer Aufführungsorte. Im Bezirksamt Wien-Meidling wurden ebenso Fremdenverkehrsfilme vorgeführt wie im Schutzhaus Rosenthal oder im Wartesaal des Wiener Südbahnhofs. ${ }^{582}$ Die Idee, Reisefilme Open Air zu zeigen, hatte man offensichtlich vom

575 Zimmermann, Industriefilme, S. 287.

576 WStLA, MA 104, Zl. K 2341, „Konzessionsansuchen Julius Maggi“, 22. Februar 1938, beigelegt Schreiben des Stadtschulrats für Wien vom 30.3.1938.

577 Zimmermann, Industriefilme, S. $286 \mathrm{f}$.

578 WStLA, MA 104, Zl. K 852, „Ankündigung. Maggi-Filmvorführung im Landegericht II:“, 19. März 1928.

579 WStLA, MA 104, Zl. K 157, „Konzessionsansuchen Zeiss-Ikon Filmvorführung im Militärkasino“, 28. März 1935 sowie ebd., Zl. K 1125, „Konzessionsansuchen Kodak, Vorträge“, 22. September 1936.

580 WStLA, MA 104, Zl. K 1675, „Bericht über die Errichtung einer Lüftungsanlage in dem im Bau befindlichen Reklamekino der städtischen Elektrizitätswerke“, Juni 1937.

581 WStLA, MA 104, Zl. K 1617, „Konzessionsansuchen Adolf J. Titze A.G., Werbe-Tonfilmvorführung in Wiener Wohnhausanlagen“, 26. September 1929.

582 WStLA, MA 104, Zl. K 2882, „Filmvortrag Urlaubreisen im Autobus im Magistratischen Bezirksamt, Wien XII.“, 2. März 1932. Ebd., Zl. K 961, „Konzessionsansuchen Filmbericht des Österreichischen Verkehrsbüros im Schutzhaus Rosenthal“, 17. Juni 1936. Ebd., Zl. K 1516, „Konzessionsansuchen, Vorführung von Filmen im Wartesaal des Südbahnhofs“, 10. März 1937. 
französischen Außenministerium übernommen, das schon 1927 „Aufnahmen aus allen möglichen Gegenden Frankreichs im Zentrum Wiens“ auf offener Straße präsentierte. ${ }^{583}$ In Wien brachte man touristisch motivierte Filme am Heldenplatz, auf der rechten Seite des Äußeren Burgtors, dar. ${ }^{584} 1934$ ließ man sich diesbezüglich mit einer Wanderkinoausrüstung auf einem Kinderspielplatz in der städtischen Gartenanlage VI (Mariahilfer Gürtel) nieder. ${ }^{585}$

Mobile Kinoeinrichtungen hatten mit den 1920er-Jahren auch in Österreich immer größere Verbreitung gefunden. Für viele Menschen bot diese neue technische Errungenschaft zudem die Möglichkeit, sich den Lebensunterhalt zu verdienen. Kriegsinvalide suchten ebenso um eine Konzession zur Vorführung von Werbefilmen an wie etwa auch alleinstehende Frauen, denen es an einer Erwerbsmöglichkeit im erlernten Beruf mangelte. ${ }^{586}$ Gleichfalls suchten Unternehmer, die sowohl Reklamestreifen herstellten als auch präsentierten, um dauerhafte Vorführgenehmigungen an (so etwa Friedrich Katz und Hans Ludwig Böhm). ${ }^{587}$ Für das Zeigen der Filme wurden die Konzessionsinhaber von den Auftraggebern (den Produktproduzenten bzw. Dienstleistungsanbietern) bezahlt. Nicht selten brachten sie beim Konzessionsgesuch vor, ausschließlich österreichische Waren bewerben $\mathrm{zu}$ wollen, womit ein positiver Entscheid forciert wurde. ${ }^{588}$

$\mathrm{Zu}$ sehen waren die Streifen in Durchhäusern, in Litfaßsäulen, in Geschäftslokalen, in Auslagen oder in mobilen Reklamefahrzeugen. Letztere wurden oftmals speziell konstruiert sowie deren Einsatz mit Lage- und Projektionsskizzen detailliert beschrieben. ${ }^{589}$ Im Herbst 1935 richtete man in der Kärntner Straße 23 sogar ein eigenes „Propaganda-Schau-Lokal“ ein, das Industrie, Gewerbe und

583 Österreichische Film-Zeitung, „Das Freilicht-Kino in Wien“, Nr. 4, 22. Jänner 1927, S. 9.

584 WStLA, MA 104, Zl. K 3102, „Österreichische Kulturfilmstelle, Vorführung von Stadt- und Landschaftsbildern aus dem österreichischen Bundesgebiet auf dem Heldenplatz“, ohne Tag/ Datum, 1932.

585 WStLA, MA 104, Zl. K 1301, „Österreichische Kulturfilmstelle, Vorführung von Filmen zur Förderung der Volkswirtschaft und des Fremdenverkehrs auf dem Kinderspielplatz in der städtischen Gartenanlage VI.“, 29. Mai 1934.

586 WStLA, MA 104, Zl. K 1701, „Konzessionsansuchen, Kriegsinvalide“, 26. Mai 1937. Ebd., Zl. K 828, „Konzessionsansuchen Ludwig Müller, Kriegsleiden“, 3. März 1928. Ebd., Zl. K 851, Konzessionsansuchen Marie Denerling, Modistin, Vorführung von Filmen in Durchhäusern“, 21. Juni 1928. Ebd., Zl. K 1215, „Konzessionsansuchen Helene Kletzka, bislang Vertreiberin von kosmetischen Produkten“, 22. Oktober 1936. Ebd., Zl. K 1421, „Konzessionsansuchen Norbert Lausch zur ständigen gewerbsmäßigen Vorführung von Werbe- und Propagandafilmen“, 23. Jänner 1937.

587 WStLA, MA 104, Zl. K 4007, „Konzessionsansuchen Hans Ludwig Böhm“, 12. Juli 1934. Ebd., Zl. K 694, „Konzessionsansuchen Dr. Friedrich Katz“, 31. Jänner 1936.

588 Vgl. u. a.: WStLA, MA 104, Zl. K 449, „Hoffmann Hermann, Konzessionsansuchen“, 19. Oktober 1935.

589 WStLA, MA 104, Zl. K 926, „Kotzian Anton, Konzession zur Vorführung von Reklamefilmen mittels eines automobilen Projektionswagens“, 11. Mai 1928. 


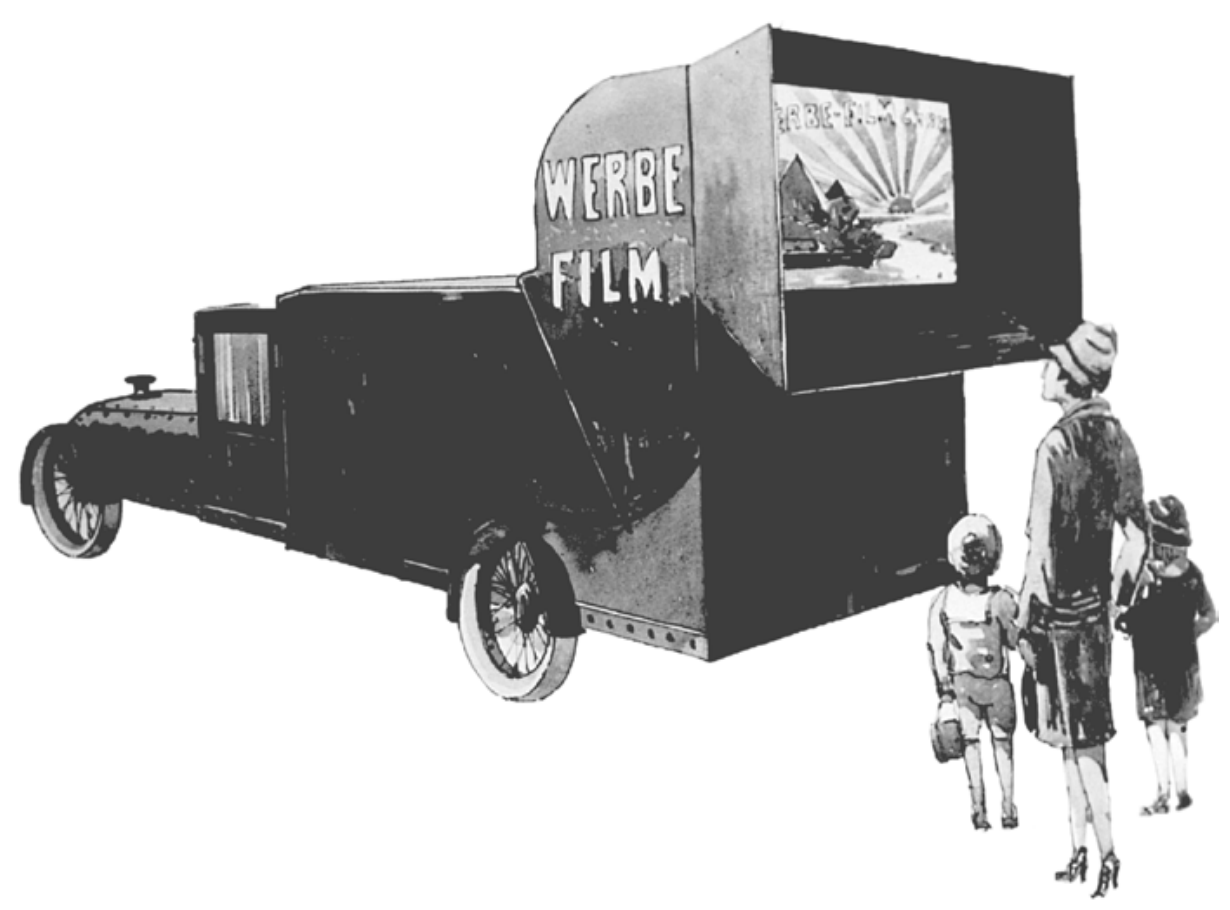

Abb. 5: Reklame-Projektionswagen, System Anton Kotzian, 1928.

Fremdenverkehr „vornehme und neuzeitliche Ausstellungsräume“ bot, in denen Werbefilme zur Aufführung kamen. ${ }^{590}$

Die Häufigkeit der Ansuchen um Vorführung von Schmalfilmen zu Reklamezwecken mobilisierte zunehmend die Gremien der österreichischen Lichtspielunternehmen. ${ }^{591}$ Besonders missfiel den Kinobetreibern, wenn einzelne Unternehmer „Wanderkonzessionen“ beantragten bzw. eine Vielzahl von Aufführungsorten bespielen wollten. Man erhob gegen Genehmigungen des Magistrats mit Hinweis auf den notwendigen „Schutz der schwer um ihre Existenz kämpfenden erwerbstätigen Kinos“ Einspruch. Die Präsentation von Reklamefilmen sollte ausschließlich im jeweiligen „eigenen Geschäftsstandort“ oder in einem „,in der Nähe hiezu gemieteten Lokal“ erfolgen. Der „Zutritt zu den Vorführungen müsse unentgeltlich“ sein. Die

590 WStLA, MA 104, Zl. K 425, „Königsberger Eduard, Konzession zur Vorführung von Reklamefilmen im Lokale der Propagandaschau“, 14. September 1935.

$591 \mathrm{Zu}$ nennen wären diesbezüglich das „Gremium der Lichtspielunternehmer Österreichs“, die „Gewerkschaft für das Bühnen-, Kino- und Filmpersonal“ sowie der „Bunde der Wiener LichtspielTheater“. 
„zulässige Anzahl gleichzeitig anwesender Zuseher wäre in angemessenem Maße zu beschränken wie auch die Meterlänge der vorzuführenden Schmalfilme“. 592

Oftmals gab das Magistrat den Vertretern der Kinounternehmen recht und verweigerte die Ausstellung einer Vorführkonzession. Ein schlagkräftiger Grund für eine Zurückweisung des Gesuchs war die Aufführung von Filmen, die nicht ausschließlich der Bewerbung von Produkten oder Dienstleistungen dienten, sondern vielmehr Kulturfilm-, Sportfilm-, Landschaftsfilm-, Spielfilm- oder Aktualitätencharakter aufwiesen. ${ }^{593}$ Davon betroffen war etwa auch die Firma „Julius Maggi Gesellschaft m.b.H.“, die neben ihren klassischen Werbestreifen auch den Reisefilm VON WIEN BIS BREGENZ zur Vorführung brachte. Dem Unternehmen wurde die Präsentation dieses Films „zum Schutz der Lichtspielbetreiber“ untersagt. Die Werbeleiter von „Maggi“ reagierten prompt und ersetzten den Reisefilm durch Diapositive mit dem gleichen Inhalt. Das Reklamekonzept, das auf der Darbietung von Werbefilmen und einem Vortrag (Letzterer nun eben mit Hilfe von Lichtbildern) basierte, blieb erhalten. Die Kinobetreiber waren mit dieser Lösung zufrieden. ${ }^{594}$

Anders verlief eine Auseinandersetzung rund um die Beantragung einer Wanderkinokonzession seitens der Firma „Persil-Gesellschaft Henkel \& Voith m.b.H.“. Gegen deren Bewilligung erhoben die Kinounternehmer aus „Konkurrenzgründen“ Einspruch. Das Unternehmen setzte sich gegen diesen Protest zur Wehr und brachte zahlreiche Argumente vor, die für die positive Beurteilung des eigenen Gesuchs sprachen. Vorerst erklärte die „Persil-Gesellschaft“, mit ihren kostenlosen Werbe- und Waschvorführungen in keinerlei Wettstreit mit dem regulären Kinoprogramm zu stehen. Man „entziehe“ somit „einem Haushalt niemals den für die Unterhaltung möglichen Betrag“. Vielmehr hätte die Firma mit ihren Präsentationen in bäuerlichen Kreisen oft erstmals deren Interesse am Tonfilm geweckt. In der Folge wären viele Persilabend-Besucher zu Kinobesuchern avanciert. Über lange Zeit habe das Unternehmen zudem seine Filme ausschließlich in Kinos zur Aufführung gebracht. Allein der Einsatz des Films WÄSCHE-WASCHEN-WOHLERGEHEN hätte den österreichischen Lichtspielbetreibern 175.173,60 Schilling an Kinomieten eingebracht. Es habe sich jedoch als notwendig erwiesen, eigene Vorführeinrichtungen zu nutzen, da die Kinobetreiber abendfüllende Werbefilme meist nur

592 WStLA, MA 104, Zl. K 673, „Hoffmann \& Co., Konzessionsansuchen, 44 Standorte“, 25. März 1936. Vgl. weiters: Ebd., Zl. K 1215, „Konzessionsansuchen Helene Kletzka, bislang Vertreiberin von kosmetischen Produkten“, 22. Oktober 1936. Ebd., Zl. K 1870, „Konzessionsansuchen der Konsumgenossenschaft Wien und Umgebung“, 10. September 1937.

593 Siehe z. B.: WStLA, MA 104, Zl. K 555, „Konzessionsansuchen für Schmalfilmvorführungen im Direktionsgebäude der städtischen Gaswerke“, 22. November 1935. Ebd., Zl. K 452, „Konzessionsansuchen Kodak“, 2. Oktober 1935. Ebd., Zl. K 673, „Hoffmann \& Co., Konzessionsansuchen, 44 Standorte“, 25. März 1936.

594 WStLA, MA 104, Zl. K 1710, „Konzessionsansuchen Maggi“, 18. November 1929. Sowie unter dem gleichlautenden Betreff: Ebd., Zl. K 1806, 3. Jänner 1930. 
nachmittags vorführen wollten. Berufstätigen Frauen, die man mit der Kampagne auch erreichen wollte, wäre es so unmöglich gewesen, die Vorführungen zu besuchen. In kleineren Orten fehle es zudem oft an einer Lichtspielstätte. Einen Anreiseweg zu einer Reklamevorstellung von mehr als 10 bis 15 Minuten könne man den Frauen aber genauso wenig zumuten wie zusätzliche Fahrtkosten. Mitunter gäbe es in einem Ort zwar ein Provinzkino, doch oftmals ohne oder mit einer sehr schlechten Tonanlage. Um die eigenen Werbefilme nun aber in bester Qualität und möglichst flächendeckend vorführen zu können, wären Wanderkinoeinrichtungen eine absolute Notwendigkeit. Umfassende Investitionen wurden hierfür bereits getätigt. Die Errichtung von Lehr-und Ausstellungsräumen käme direkt der österreichischen Wirtschaft zugute und schaffe zudem Arbeitsplätze. Das Gleiche gelte für den Wanderkino- und Ausstellungsbetrieb. Auch habe die „PersilGesellschaft“ mit einem Kostenaufwand von 300.000 Schilling einen großen Werbefilm (WÄSCHE-WASCHEN-WOHLERGEHEN) in Österreich mit heimischem Personal hergestellt und auch damit die alpenländische (Film-)Wirtschaft direkt unterstützt. Ein Ablehnen der Wanderkinolizenz hätte den Verlust zahlreicher Investitionen und Arbeitsplätze zur Folge und würde die Firma in ihrem ökonomischen Fortkommen behindern. ${ }^{595}$ Unterstützt wurde Persil in dieser Frage auch von den österreichischen Kurzfilmherstellern, die sich ebenfalls Aufträge erhofften und somit in der Erteilung einer Wanderkonzession für das Unternehmen auch einen Gewinn für die heimische Filmwirtschaft erkennen wollten. ${ }^{596}$ Der Magistrat schloss sich der Argumentation der „Persil-Gesellschaft“ und der Kurzfilmhersteller an und erteilte angesichts der ,wirtschaftlichen Bedeutung und des Rufs der Firma“ sowie im Hinblick „auf die Schaffung neuer Arbeitsplätze“ einen positiven Bescheid. ${ }^{597}$

Der Kampf um die ökonomische Existenz war innerhalb der Filmbranche nicht nur auf die Kinobetreiber beschränkt. Auch die Produzenten von Werbe-,Kultur-und Bildungsfilmen reagierten auf den wirtschaftlichen Druck der Zwischenkriegszeit und setzten Zeichen, um ihre Position zu sichern und zu festigen.

595 WStLA, MA 104, Zl. K 186, „Konzessionsansuchen Persil“, April/Mai 1935.

596 ÖStA, AdR, BMHV, 581c, Grz. 100525-WPA/35, Zl. 110231, „Schreiben Verband der Kurzfilmhersteller an Handelsministerium bezüglich Henkel \& Voith Wien, 29. Oktober 1935.

597 WStLA, MA 104, Zl. K 186, „Konzessionsansuchen Persil“, April/Mai 1935. 